\title{
Delimitación del perfil competencial inclusivo necesario en la formación inicial en los grados de educación primaria
}

\author{
Delimitation of the inclusive competency profile necessary \\ in initial training in elementary education grades
}

\author{
Xènia Garcia-Vallès \\ Investigadora, Centre d'Estudis i Recerca per a una Societat Inclusiva \\ Universidad Autonóma de Barcelona \\ Barcelona, España \\ xenia.garcia@uab.cat
}

Recibido - Received: 28 / 02 / $2020 \quad$ Corregido - Revised:08 / 05 / 2020 Aceptado - Accepted:01 / 06 / 2020

\author{
DOl: https://doi.org/10.22458/ie.v22i32.2830 \\ URL: https://revistas.uned.ac.cr/index.php/innovaciones
}

\begin{abstract}
Resumen: Uno de los factores que inciden en el desarrollo de procesos inclusivos en los centros escolares es la formación inicial del profesorado en el campo de la educación inclusiva. La universidad, como centro formador, ha optado por combinar los dos modelos existentes: un modelo más transversal de la formación sobre la educación inclusiva y otro más específico. La elección de los modelos formativos está relacionada con la concepción que se tenga sobre la educación inclusiva. No obstante, sería relevante identificar cuáles son las competencias necesarias para que el futuro profesorado pueda implementar procesos inclusivos en las escuelas. En este sentido, el artículo tiene como objetivo identificar las competencias en educación inclusiva (CEI) claves para la formación inicial (FI) en los grados de educación primaria. A través del método Delphi, en el que han participado expertos y profesionales del ámbito de la educación inclusiva, los resultados apuestan por una formación universitaria en el Grado de Educación Primaria, donde prevalezcan competencias transversales relacionadas con el trabajo en equipo, la comunicación, la dinamización de los apoyos, la colaboración, el liderazgo, la resolución de conflictos y una actitud crítica y positiva, que permita alcanzar procesos educativos inclusivos.
\end{abstract}

Palabras clave: Competencias del profesorado, formación inicial del profesorado, enseñanza superior, planificación de la educación, inclusión, innovación educativa

Summary: One of the factors that influence the development of inclusive processes in schools is the initial teacher training in the field of inclusive education. The university, as a training center, has chosen to combine the two existing models: a more transversal model of training on inclusive education and a more specific one. The choice of training models is related to the conception of inclusive education. However, it would be relevant to identify what are the necessary competencies so that future teachers can implement inclusive processes in schools. In this sense, the article aims to identify the key skills in inclusive education (CEl competences for inclusive education - acronym in Spanish) for initial training (FI - acronym in Spanish) in elementary education grades. Through the Delphi method, in which experts and professionals from the field of inclusive education have participated, the results are committed to university training in the Elementary Education Degree, where transversal competences related to teamwork, communication, energization of supports, collaboration, leadership, conflict resolution and a critical and positive attitude that allows inclusive educational processes to be achieved

Key Words: Teacher competences, initial teacher training, higher education, educational planning, inclusion, educational innovation 


\section{INTRODUCCIÓN}

En la actualidad, se considera "educación de calidad", aquella que promueve escuelas inclusivas y verdaderamente democráticas (González-Gil et ál., 2013; Jiménez, 2012; UNESCO, 2016).

Un primer aspecto, necesario de tener en cuenta para que estas sean democráticas, se relaciona con el derecho al acceso a los diferentes centros escolares por parte de todos los colectivos, sin etiquetar ni excluir al futuro alumnado por sus características. Se observa, desde la literatura científica, que existen esfuerzos constantes para argumentar y/o demostrar los beneficios relacionados con la escolarización del alumnado diverso en escuelas ordinarias. Esto se debe a que aún existen barreras que dificultan lograr un acceso igualitario y democrático (Blanco y Duk, 2019; Forgiony-Santos, 2019; Hardy y Woodcock, 2015; Pujolàs, 2010).

Es el caso de la Agency for Special Needs and Education (2018), en su informe sobre Pruebas de relaciones entre la educación inclusiva y la educación social, afirma, que existe una relación directa entre los niños que han recibido una educación inclusiva en centros ordinarios, y su capacidad de lograr un mayor grado de autonomía en el ámbito laboral, familiar y social.

Un segundo aspecto, considerado por la comunidad científica como imprescindible para la consecución de escuelas inclusivas, se relaciona con el papel que desempeñan los diferentes agentes de la comunidad educativa (familias, alumnado, profesorado, etc.). Entre los diferentes agentes, el profesorado se considera una pieza fundamental a la hora de desarrollar procesos educativos inclusivos así como prácticas inclusivas (Duk y Murillo, 2016; García, Herrera-Seda y Vanegas, 2018; Luzón y Montes, 2018). La capacidad del profesorado para poder atender la diversidad en los centros escolares mantiene una relación directa con la denominada pedagogía inclusiva, la cual se basa en el reconocimiento que el profesorado con su práctica diaria puede reducir las desigualdades y mejorar la calidad final educativa (Florian, 2015).

Así mismo, la realidad se aleja de la situación ideal, ya que, ni el profesorado recibe una formación de calidad (Perlado, Muñozy Torrego, 2019) ni todos los niños realizan su escolaridad obligatoria en centros ordinarios. La Organización de Naciones Unidas, más concretamente su Comité sobre los Derechos de las Personas con Discapacidad (2018), publicó una investigación ( CRPD/C/18/R.23), sobre la actualidad de la educación inclusiva en España. Se deriva del informe que en España sigue siendo legal dejar fuera al alumnado de la escuela ordinaria y la clasificación de nuestro sistema educativo como excluyente. En el último plan en Cataluña, Ilamado Plan para la reducción del fracaso escolar en Cataluña (2012-2018), se concluye que es necesaria una mejora de la competencia profesional de los docentes a través del impulso de la formación inicial y continua y su adecuación a las necesidades educativas específicas del alumnado.

Aunque, ha habido mejoras, una de las más importantes el apoyo de un marco legal que obliga a las escuelas a ser inclusivas, no siempre se transfieren en las prácticas de los diferentes centros escolares. El profesorado puede tener un papel destacado en la mejora de los procesos inclusivos en las escuelas. Para que esto ocurra, se requiere de una formación inicial de calidad.

\section{Formación inicial en educación inclusiva}

En relación con la formación de competencias profesionales en educación inclusiva, específicamente en el grado de educación primaria, la implementación del Plan Bolonia en las universidades europeas ha marcado tendencias y ha influido en esta. Aparte del cambio de paradigma sobre el conocimiento y sus pertinentes efectos, en el perfil competencial del futuro profesorado (Mas-Torelló y Olmos-Rueda, 2016), la implementación del Espacio Europeo de Educación Superior ha supuesto una reforma de los planes de estudio de magisterio, en el cual se ha pasado de un modelo dual de formación a un modelo 
integrado de formación (Izuzquiza, Echeita, y Simón, 2015).El modelo integrado permite la posibilidad de ofrecer dos tipologías de formación en CEI. Una primera, en la cual la formación de las CEI se realiza a través de módulos o asignaturas específicas, y una segunda, en la que se ofrece una formación transversal en educación inclusiva. La decisión sobre qué tipología de formación implementarse relaciona con la concepción que se tenga sobre educación inclusiva. No existe actualmente un consenso sobre cuál modelo se debe aplicar, más bien, existen opiniones contrarias en la literatura actual. En primer lugar, defensores de un modelo transversal e integral (Leiva y Gómez, 2015; European Comission, 2017) yen segundo lugar, estarían los que consideran que la transversalidad puede ser peligrosa por el hecho de que, en general, las responsabilidades compartidas terminan siendo responsabilidades de nadie(Izuzquiza et ál., 2015). Así pues, sin ningún consenso y centrándonos de nuevo en la Fl en CEl, existe un aspecto común en los dos modelos y es que ambos requieren de una delimitación competencial. Por tanto, se hace imprescindible reflexionar sobre cuál perfil competencial inclusivo requiere nuestro contexto y definir cuál perfil competencial inclusivo "ideal" tendría que dominar el futuro profesorado, una vez finalizados los estudios del Grado en Educación Primaria.

Se cree oportuno analizar antes qué se entiende por competencia, pues no existe un acuerdo ni univocidad de cómo es entendida. Diferentes autores han centrado sus trabajos en recopilar las definiciones existentes y discernir en ellas características reiterativas y claves del propio concepto. Mas (2009, p. 194) recopila y sintetiza múltiples definiciones y análisis de las características en común citadas ya por otros autores, destacando las siguientes: las competencias se vinculan a un contexto determinado; se han de poder aplicar relacionándose de manera directa con el desarrollo profesional y la resolución de problemas; responden al dinamismo y reconstrucción pudiendo variar así su utilidad, orientación y exigencia según el contexto y el momento; han de ejercer la función de transferencia y resolución de problemas; implican una determinada manera de procesar la información; permiten actuar de un modo diferenciado e idóneo delante de las diferentes situaciones; se consideran complejas, porqué sostienen esquemas de pensamiento y a la vez integradoras de los mismos esquemas y/o saberes $y$, finalmente, responden a la característica de interdependencia, ya que no se puede hablar de competencias aisladas, sino transversales que comparten conocimientos, habilidades y actitudes.

La definición de competencia emitida por la Comisión Europea (2013): "Las competencias corresponden a una combinación de conocimientos, habilidades, valores y actitudes "prioriza, en este caso, el concepto de transversalidad; es el principio con el que nos identificamos. La Fl, en CEl, ha de responder a un trabajo coordinado de las diferentes asignaturas de los Grados de Educación Primaria, el cual permita también una relación constante entre conocimientos, habilidades y actitudes. Los conocimientos, desde una lógica integrante, sirven para adquirir y/o desarrollar dichas competencias. Las habilidades requieren de una reflexión y elección respaldadas por unos conceptos, actitudes y valores. Por último, los "valores y actitudes" implican una disposición a actuar (actitud) de acuerdo con los valores. Son considerados como indispensables en la formación inicial y continua de competencias inclusivas ya que, sin actitud y valores inclusivos, se hace difícil la transferencia de procesos inclusivos en los centros escolares y sus aulas (Darling-Hammond y Lieberman, 2013; Novo-Corti, Muñoz-Cantero y Calvo-Porral, 2011; Srivastava, de Boer y Pijl, 2015).

Las competencias, que han de configurar el perfil competencial en educación inclusiva, han de aportar conocimientos teóricos y procedimientos metodológicos de un ámbito profesional o titulación concreta. Estas competencias se construyen teniendo en cuenta el ámbito académico así como el ámbito profesional (Prades, 2005). Para ello, y en lo relativo al ámbito académico, nos basaremos en los trabajos realizados desde una perspectiva internacional sobre la formación inicial en CEl (Alegre, 2010; Echeita, 2015; Fernández, 2012; García, 2005; Grande y González, 2015; Liasidou y Antoniou, 2015; Moriña, Cortés, y Molina, 2015; Rodríguez, 2019; Sales, 2006; Van Laarhoven, Munk, Lynch, Bosma, y Rouse, 2007). Se han detectado similitudes entre la tipología de competencias delimitadas, pudiéndose clasificar en una serie de ámbitos que, de una manera general, todos los autores repiten. Los ámbitos corresponden a: trabajo en equipo y cooperación; valores y ética; pedagógico-didáctico; liderazgo; tecnológico; evaluación y personales. 
Existen otras coincidencias entre los autores vinculadas a las competencias consideradas inclusivas que debería poseer el profesorado y relacionadas con los siguientes aspectos:

a. Capacidad de comunicación con todos los agentes educativos y de realizar un trabajo colaborativo; valores y actitudes positivos hacia la diversidad y un claro compromiso hacia una mejora de todo el alumnado;

c. dominio de las diferentes técnicas pedagógicas y psicológicas para el trabajo concreto en el aula;

d. rasgos de liderazgo, destacando su capacidad para tomar decisiones;

e. capacidad de usar y reconocer la tecnología, así como su adaptación a un sistema de aprendizaje universal y una actitud constante de mejora y crítica profesional y personal, con la finalidad de ofrecer un bienestar en el quehacer diario del centro escolar.

A continuación, se presenta una tabla con las competencias seleccionadas, sus autores y clasificadas en sus respectivos ámbitos:

TABLA 1

Relación de las competencias en educación inclusiva seleccionadas y sus autores, clasificadas en sus respectivos ámbitos

\begin{tabular}{|c|c|c|}
\hline Ámbitos & Competencias seleccionadas & Autores \\
\hline \multirow{6}{*}{$\begin{array}{l}\text { Trabajo } \\
\text { en equipo } \\
\text { y cooperación }\end{array}$} & Realimentarse constantemente con el alumnado. & (García, 2005) \\
\hline & Colaborar constantemente y trabajar en equipo. & (Echeita, 2015) \\
\hline & Colaborar entre redes y profesionales. & (Liasidou y Antoniou, 2015) \\
\hline & Conformar equipos de apoyo y redes de apoyo institucional. & (Alegre, 2010) \\
\hline & $\begin{array}{l}\text { Establecer una relación de confianza con las familias y favorecer la comunicación. Informar } \\
\text { sobre la evolución del alumnado. Colaborar con otros agentes educativos. Diseñar activi- } \\
\text { dades de trabajo colaborativo y resolución de problemas. }\end{array}$ & (Fernández, 2012) \\
\hline & $\begin{array}{l}\text { Trabajar colaborativamente, flexibilidad y adaptabilidad. Habilidades para favorecer el dia- } \\
\text { logo y la reciprocidad con las familias. }\end{array}$ & (Grande y González, 2015) \\
\hline \multirow{6}{*}{ Valores y ética } & Animar y motivar a todo el alumnado. Compromiso y actitud positiva hacia la diversidad. & (García, 2005) \\
\hline & Apoyo, altas expectativas y persecución del éxito para todos. Valorar la diversidad. & (Echeita, 2015) \\
\hline & Justicia Social. & (Liasidou y Antoniou, 2015) \\
\hline & Ayudar, dinamizar y motivar al estudiante. & (Fernández, 2012) \\
\hline & Actitud positiva hacia la diversidad. & (Van Laarhoven et. ál., 2007) \\
\hline & Creer que todos los alumnos pueden aprender y mejorar su aprendizaje. & (Liasidou y Antoniou, 2015) \\
\hline \multirow{7}{*}{$\begin{array}{l}\text { Pedagógico- } \\
\text { didáctico }\end{array}$} & $\begin{array}{l}\text { Acompañar, corregir, avaluar, individualizar el aprendizaje y planificación. Diagnosticar di- } \\
\text { ficultades del aprendizaje. Conocer diferentes recursos y aplicarlos. }\end{array}$ & (García, 2005) \\
\hline & Trabajar el aprendizaje emocional. & (Echeita, 2015) \\
\hline & Conocer estrategias psicopedagógicas para atender al alumnado. & (Liasidou y Antoniou, 2015) \\
\hline & $\begin{array}{l}\text { Identificar las necesidades y conflictos de la interacción enseñanza-aprendizaje con estu- } \\
\text { diantes con NEE. Incorporar modificaciones en el currículo, que lo aparten le menos posi- } \\
\text { ble de la programación curricular. }\end{array}$ & (Alegre, 2010) \\
\hline & $\begin{array}{l}\text { Tutorizar, comunicar, gestionar metodologías activas, acomodar la enseñanza y adecuar } \\
\text { los materiales. }\end{array}$ & (Fernández, 2012) \\
\hline & Conocer el currículo y su didáctica. Saber realizar el diagnóstico inicial. & (Van Laarhoven et. ál, 2007) \\
\hline & $\begin{array}{l}\text { Aplicar estrategias en el aula para atender la diversidad, planificar, desarrollar y avaluar } \\
\text { propuestas. Adaptar materiales. Conocer los recursos psicopedagógicos. Favorecer el am- } \\
\text { biente inclusivo. }\end{array}$ & (Grande y González, 2015) \\
\hline
\end{tabular}



toma de decisiones en un marco común.

Evaluación

Realizar evaluaciones con capacidad crítica.

(Liasidou y Antoniou, 2015)

Llevar a cabo valoraciones de los potenciales del alumno y de sus contextos.

(Alegre, 2010)

Llevar a cabo valoraciones de los potenciales del alumno y de sus contextos.

(Alegre, 2010)

Tecnológico

Usar y conocer las tecnologías necesarias.

(Van Laarhoven et. ál, 2007)

Conocer los recursos TIC y su adaptación universal

(Grande y González, 2015)

Fomentar la formación profesional y personal al profesorado constante.

(Echeita, 2015)

Identificar y desarrollar estrategias innovadoras.

(Alegre, 2010)

Personal Escuchar de forma activa y empáticamente. Ser paciente y tolerante, fiel a sus valores, puntual, responsable, respeta las reglas y normas, honesto, ético, modelo, asume compromisos y tareas.

(Fernández, 2012)

Tener una actitud positiva y capacidad de motivación.

(Liasidou y Antoniou, 2015)

Fuente: Elaboración propia (2020).

\section{MATERIALES Y MÉTODOS}

Con el fin de identificar las CEI teniendo en cuenta el ámbito académico y profesional, se ha aplicado la técnica Delphi a un colectivo de expertos en la materia. Tal y como apuntan Gil-Gómez y Pascual-Ezama (2012), esta metodología es de gran utilidad para obtener validez de contenido. Se basa en dos principios fundamentales: la inteligencia colectiva y la participación anónima.

Se ha seguido una estructura metodológica para la aplicación de la técnica Delphi basada en: 1) selección de los expertos; 2) calidad de los expertos; 3) proceso dividido en rondas; 4) consenso y estabilidad.

\section{Selección de los expertos}

La principal dificultad de su selección se relaciona con que la palabra "experto" puede tener diferentes connotaciones (Cabero e Infante, 2014). Es imprescindible, delimitar criterios que nos permitan seleccionar a los expertos. En la presente investigación, los criterios han sido los siguientes:

TABLA 2

Criterios para la selección de los expertos

\section{Criterios para la selección de los expertos}

Voluntad de participar en todo el proceso

Nivel académico

Institución en la que trabajan

Años de experiencia profesional

Categoría profesional

Experiencia relacionada con la inclusión educativa y/o formación inicial del profesorado

Fuente: Elaboración propia (2020). 
En cuanto al número de expertos, no existe todavía un acuerdo científico al respecto. Se ha defendido que, según la naturaleza de la técnica, se puede realizar una aproximación del número si este es un grupo homogéneo (de entre 10-15 expertos). Si es heterogéneo (estudios internacionales), se pueden requerir hasta cientos de expertos (Skulmoski, Hartman y Krahn, 2007). Nuestro estudio corresponde a una muestra homogénea, por lo tanto, y analizando lo expuesto anteriormente, la selección final ha sido de diez expertos. Los expertos escogidos fueron los siguientes:

TABLA 3

Tipología de expertos participantes en el estudio

\begin{tabular}{llc}
\multicolumn{1}{c}{ Tipología expertos } & \multicolumn{1}{c}{ Ámbito } & Participantes \\
Docentes/investigadores & Universitario & 3 \\
Docentes & $\begin{array}{l}\text { Centros educativos } \\
\text { Equipo de Asesoramiento }\end{array}$ & 3 \\
$\begin{array}{l}\text { Otros profesionales } \\
\text { del ámbito educativo }\end{array}$ & $\begin{array}{l}\text { Psicopedagógico, inspección, } \\
\text { consorcio de educación. }\end{array}$ & 4 \\
TOTAL & & 10 \\
\hline
\end{tabular}

Fuente: Elaboración propia (2020).

\section{Calidad}

Diferentes autores hablan del "coeficiente de competencia experta" (Cabero y Barroso, 2013) para la correcta evaluación de su pericia. El cálculo de pericia de los diez expertos participantes correspondió a:

TABLA 4

Cálculo de pericia de los expertos participantes

\begin{tabular}{cc} 
Participante experto & $\begin{array}{c}\text { Coeficiente } \\
1\end{array}$ \\
2 & 0,95 \\
3 & 1,0 \\
4 & 0,95 \\
5 & 0,9 \\
6 & 0,9 \\
7 & 0,9 \\
8 & 0,8 \\
9 & 0,85 \\
10 & 0,85 \\
\multicolumn{2}{c}{ Fuente: Elaboración propia $(2020)}$.
\end{tabular}

La media del índice de competencia de todos los expertos consultados (Compa) correspondió a $\mathbf{0 , 8 8 ;}$ por tanto, eran expertos de alta influencia. Por otro lado, se valoró la motivación de los participantes, todos aseguraron estar dispuestos a iniciar y finalizar el estudio. Se obtuvieron valoraciones (en una escala del 1 al 10), correspondientes a valores entre el 9 y el 10 ( 7 expertos marcaron el número 9 y 3 marcaron el número 10), obteniendo así un valor medio de 9,3 en una escala del 0 al 10. 


\section{Proceso dividido en rondas}

En relación con el número de rondas aplicadas, Steurer (2011) evidencia que la mayoría de las aplicaciones de la técnica Delphi se desarrolla en dos rondas y a veces en tres. El proceso seguido en la presente investigación ha consistido en la aplicación de tres rondas a los expertos ya mencionados. Siguiendo las recomendaciones de Hung et ál. (2008, p. 197), se acordó realizar una primera ronda, la cual perseguía el objetivo de obtener el máximo de información posible, para poder así lograr consenso en las siguientes dos rondas. Así pues, para la primera ronda se elaboró un cuestionario inicial a partir del análisis documental realizado (artículos científicos y guías docentes del grado de primaria de todas las universidades públicas catalanas), en el cual los expertos podían ver diferentes competencias clasificadas en ámbitos. Los ámbitos presentados a los expertos fueron un total de siete ámbitos correspondientes a: trabajo en equipo y cooperación; valores y ética; pedagógico-didáctico; liderazgo; tecnológico; evaluación y personal.

\section{Consenso y estabilidad}

Se han consensuado criterios de elaboración y escrutinio con el objetivo de obtener coherencia y validez en los resultados de cada una de las rondas. Se persigue la máxima estabilidad en los resultados obtenidos en la tercera y última ronda de la técnica Delphi aplicada.

TABLA 5

Indicaciones para los expertos en cada ronda y criterios aplicados para la delimitación de las competencias en cada una de las rondas

\begin{tabular}{|c|c|c|c|c|}
\hline \multirow[b]{2}{*}{ Rondas } & \multirow{2}{*}{$\begin{array}{l}\text { Criterios dirigidos } \\
\text { a los expertos }\end{array}$} & \multicolumn{3}{|c|}{ Cálculos realizados } \\
\hline & & & Criterios & $\begin{array}{c}\text { Porcentajes para cada } \\
\text { grado de acuerdo }\end{array}$ \\
\hline \multirow{4}{*}{ Primera } & \multirow{4}{*}{$\begin{array}{l}\text { Modificar, añadir, validar o eli- } \\
\text { minar las diferentes competen- } \\
\text { cias y ámbitos. }\end{array}$} & Modificar & $\begin{array}{l}\text { Grado de acuerdo alto y medio (se } \\
\text { incorporan las diferentes compe- } \\
\text { tencias en la segunda ronda). }\end{array}$ & \multirow{4}{*}{$\begin{array}{l}=+50 \% \text { : Grado de acuerdo } \\
\text { alto } \\
29 \% \text { a } 50 \%: \text { Grado de } \\
\text { acuerdo medio } \\
29 \% \text { o }-29 \% \text { : Grado de } \\
\text { acuerdo bajo }\end{array}$} \\
\hline & & Añadir & $\begin{array}{l}\text { Siempre (Se añaden todas las com- } \\
\text { petencias con la intención de que } \\
\text { los expertos puedan leer todas las } \\
\text { propuestas en la segunda ronda). }\end{array}$ & \\
\hline & & Validar & Grado de acuerdo alto y medio & \\
\hline & & Eliminar & Grado de acuerdo alto y medio & \\
\hline \multirow[b]{2}{*}{ Segunda } & \multirow{2}{*}{$\begin{array}{l}\text { Priorizar (escala de } 1 \text { al } 5 \text { ) las } \\
\text { competencias resultantes de } \\
\text { la primera ronda y eliminar o } \\
\text { validar todas aquellas compe- } \\
\text { tencias/ ámbitos añadidos en } \\
\text { la primera ronda por los otros } \\
\text { informantes. }\end{array}$} & $\begin{array}{l}\text { Priorizar las compe- } \\
\text { tencias resultantes de } \\
\text { la primera ronda. }\end{array}$ & Grado de acuerdo alto & \multirow{2}{*}{$\begin{array}{l}=+50 \%: \text { Grado de acuerdo } \\
\text { alto } \\
29 \% \text { a } 50 \%: \text { Grado de } \\
\text { acuerdo medio } \\
29 \% \text { o }-29 \%: \text { Grado de } \\
\text { acuerdo bajo }\end{array}$} \\
\hline & & $\begin{array}{l}\text { Eliminar o validar to- } \\
\text { das las competencias } \\
\text { añadidas en la prime- } \\
\text { ra ronda por los ex- } \\
\text { pertos participantes }\end{array}$ & Grado de acuerdo alto & \\
\hline Tercera & $\begin{array}{l}\text { Se construyó un último cuestio- } \\
\text { nario con las competencias va- } \\
\text { lidadas de la primera y segunda } \\
\text { ronda, divididas por ámbitos. Se } \\
\text { pidió a los expertos que señala- } \\
\text { ran las competencias más rele- } \\
\text { vantes para ellos. }\end{array}$ & $\begin{array}{l}\text { Marcar con el sím- } \\
\text { bolo "V" las com- } \\
\text { petencias que se } \\
\text { considera que han de } \\
\text { ser priorizadas. }\end{array}$ & Grado de acuerdo alto & $\begin{array}{l}=+40 \%: \text { Grado de } \\
\text { acuerdo alto } \\
\text { 19\% a } 40 \%: \text { Grado de } \\
\text { acuerdo medio } \\
\text { 19\% o -19\%: Grado de } \\
\text { acuerdo bajo }\end{array}$ \\
\hline
\end{tabular}

Fuente: Elaboración propia (2020). 


\section{RESULTADOS Y DISCUSIÓN}

En referencia a los ámbitos, no hubo ninguna modificación por parte de los expertos, así que se mantuvieron igual que los originales. En el ámbito de competencias personales, existieron desacuerdos en su modificación, ya que algunos expertos consideraban que, en el momento de acceder a los estudios superiores, estas ya tendrían que estar consolidadas.

La evolución, en cuanto al número de competencias, fue ascendente de la primera a la segunda ronda hasta delimitarse en la tercera ronda, un total de 51 competencias divididas en siete ámbitos. Los ámbitos ordenados de más a menos competencias finales (competencias resultantes de la tercera ronda), dentro de cada ámbito, corresponde a: 1) competencias personales, 2) pedagógico-didácticas, 3) trabajo en equipo y cooperación 4) evaluación, 5) valores y ética y 6) liderazgo y 7) tecnológico. Se presenta a continuación una tabla con las competencias finales por cada una de las rondas y ámbitos:

TABLA 6

Número de competencias en las diferentes rondas y ámbitos

\begin{tabular}{lccc}
\multicolumn{1}{c}{ ÁMBITOS } & $\begin{array}{c}\text { Número } \\
\text { Competencias } \\
\text { 1a Ronda }\end{array}$ & $\begin{array}{c}\text { Número } \\
\text { Competencias } \\
\text { 2a Ronda }\end{array}$ & $\begin{array}{c}\text { Competencias } \\
\text { 3a Ronda }\end{array}$ \\
\hline Competencias personales & 20 & 21 & 12 \\
Pedagógico-didácticas & 13 & 15 & 11 \\
Trabajo en equipo y cooperación & 6 & 18 & 9 \\
Evaluación & 4 & 12 & 7 \\
Valores y ética & 10 & 10 & 5 \\
Liderazgo & 3 & 8 & 4 \\
Tecnológico & 3 & 5 & 3 \\
TOTAL & 59 & 89 & 51 \\
\hline
\end{tabular}

Fuente: Elaboración propia (2020).

Después de la aplicación de la tercera y última ronda Delphi, las competencias resultantes fueron las siguientes: 
TABLA 7

Ámbitos y competencias resultantes de la técnica Delphi aplicada a los expertos

\section{1. Ámbito trabajo en equipo y cooperación:}

1.1. Potenciar el trabajo colaborativo con las familias para favorecer la creación de entornos inclusivos.

1.2. Trabajar con los compañeros/as y con otros servicios de apoyo a la inclusión.

1.3. Colaborar con los diferentes sectores de la comunidad educativa y el entorno social.

1.4. Coordinar las intervenciones educativas de diferentes docentes que intervienen en los/las mismos/as alumnos/as o grupos de alumnos/as.

1.5. Contribuir a crear una cultura inclusiva de centro.

1.6. Favorecer procesos de reflexión y trabajo conjuntos que reconozcan los conocimientos y diferentes trayectorias profesionales.

1.7. Compartir utensilios, materiales, ayudas y planificaciones con los compañeros/as.

1.8. Favorecer procesos de identificación y análisis de las barreras y apoyos que dificultan y favorecen el aprendizaje y participación de todos los estudiantes.

1.9. Promover la colaboración con los diferentes profesionales y familias.

\section{2. Ámbito valores y ética}

2.1. Favorecer la creencia que todo el alumnado puede progresar incorporando altas expectativas en el alumnado y sus familias.

2.2. Promocionar valores vinculados al respeto, aprecio y consideración humana en todos sus aspectos.

2.3. Evitar los procesos de exclusión en todo el alumnado.

2.4. Analizar críticamente el trabajo personal para la mejora del desarrollo profesional.

2.5. Fomentar la convivencia aplicando técnicas de mediación en la resolución de conflictos, para contribuir en la resolución pacífica.

\section{3. Ámbito pedagógico-didáctico}

3.1. Planificar situaciones educativas en las cuales el alumnado con diferentes capacidades y diferentes ritmos de aprendizaje pueda participar y aprender.

3.2. Diseñar entornos accesibles.

3.3. Planificar acciones educativas significativas para todo el alumnado.

3.4. Aplicar varias estrategias de intervención basándose en los diferentes tipos de apoyos existentes.

3.5. Promover el aprendizaje cooperativo entre el alumnado.

3.6. Aplicar los conocimientos propios de la psicopedagogía para favorecer la inclusión en el aula. (Conocimiento características NEE, estrategias de clima del aula, conocimiento del sistema DUA, de los tipos de inteligencias....).

3.7. Identificar las capacidades del alumnado.

3.8. Comunicar a las familias y al alumnado las capacidades del alumnado detectadas.

3.9. Identificar las carencias formativas de la escuela.

3.10. Buscar estrategias que favorezcan la formación continuada.

3.11. Aplicar las técnicas de investigación en el aula para mejorar las prácticas inclusivas.

4. Ámbito liderazgo

4.1. Liderar procesos de colaboración con las familias para contribuir a la creación de redes de apoyo favorecedoras de los procesos de inclusión educativa y social.

4.2. Potenciar la creación de un clima de centro inclusivo, haciendo que todo el mundo se sienta aceptado y reconocido.

4.3. Liderar propuestas de planificación conjunta con todos los agentes que intervienen en los procesos de E-A.

4.4. Liderar entornos educativos fundamentados en los principios de educación inclusiva.

\section{5. Ámbito tecnológico}

5.1. Utilizar tecnologías de la comunicación e información para facilitar el acceso y la participación de todo el alumnado.

5.2. Promover trabajos de sistematización y análisis de iniciativas y experiencias con las TAC que sean favorecedoras de la inclusión.

5.3. Fomentar el uso de dispositivos digitales para compartir conocimientos con los compañeros/se construyendo y haciendo escuela inclusiva. 


\section{6. Ámbito evaluación}

6.1. Participar en actividades de evaluación compartida de la propia docencia y la de otros (observaciones, otros docentes a la propia aula, en otras aulas) para identificar barreras y realizar propuestas de mejora.

6.2. Favorecer la colaboración de las familias en los procesos de evaluación del alumnado con dificultades, aportando información sobre su actuación en otros contextos.

6.3. Identificar los propios puntos fuertes y puntos débiles.

6.4. Diseñar acciones para la mejora.

6.5. Hacer uso de instrumentos que ayuden a revisar las culturas, políticas y las prácticas inclusivas del centro educativo.

6.6. Favorecer la participación del alumnado para la mejora del centro.

6.7. Favorecer que todo el alumnado participe activamente de su evaluación

\section{7. Ámbito competencias personales}

7.1. Actuar teniendo en cuenta que los docentes son un modelo para el alumnado.

7.2. Ejercer escucha activa con el alumnado.

7.3. Buscar otras maneras de actuar (innovar) que hagan posible que todos los niños puedan sentirse una parte importante de la escuela en algún momento de sus vidas.

7.4. Buscar activamente la colaboración con otros miembros de la comunidad educativa.

7.5. Actuar de manera autónoma y reflexiva.

7.6. Comunicar con empatía.

7.7. Demostrar interés por el potencial de todo el alumnado.

7.8. Tener una actitud de ayuda a los compañeros/se docentes que lo necesiten.

7.9. Tener actitud positiva y constructiva ante la vida y de curiosidad hacia los otros, las diferentes culturas y formas de vida.

7.10. Ser responsable.

7.11. Expresar las emociones de una manera apropiada.

7.12. Tener una actitud activa hacia la formación permanente.

Fuente: Elaboración propia (2020).

Las competencias y ámbitos anteriores (tabla 7) corresponden a las competencias en educación inclusiva (CEI) claves para la formación inicial (FI) en los grados de educación primaria.

Existen algunos resultados de la presente investigación que coinciden con la literatura ya existente y refuerzan, por tanto, el perfil competencial en educación inclusiva que debería poseer el futuro profesorado.

En primer lugar, las personas expertas (de nuestro estudio) han apuntado por encima de otras competencias aquellas relacionadas con el trabajo en equipo y cooperación. Este resultado coincide con otros autores en cuyos trabajos ya habían destacado también dichas competencias (Alegre, 2010; Fernández, 2013; Grande y González, 2015; Liasidou y Antoniou, 2015; Pujolàs, 2008). Recientemente, el estudio de Perlado et ál. (2019) concluye la necesidad de mejora de la formación del profesorado en programación y organización del trabajo cooperativo en el aula para el perfeccionamiento de las prácticas inclusivas.

En segundo lugar, existen coincidencias correspondientes a la unanimidad de la importancia del dominio por parte del profesorado en competencias relacionadas con la pedagogía como: planificar, trabajar el currículo de manera flexible, individualizar el aprendizaje, adaptar materiales, evaluar y la psicopedagogía (Grande y González, 2015; Liasidou y Antoniou, 2015; Van Laarhoven et ál., 2007).

En tercer lugar, y en lo que se refiere al ámbito tecnológico, los resultados nos indican la prioridad en este ámbito de eliminar barreras para permitir el acceso a las tecnologías y a reflexionar sobre el diseño y la aplicación, teniendo en cuenta que sean verdaderamente inclusivos, estos resultados coinciden con los de Cabero y Ruiz-Palmero (2017). 
En relación con el ámbito de la evaluación, es necesario según las personas expertas, involucrar a todos los agentes educativos (familias, alumnado, profesorado, entidades educativas, entre otros) para que sean activos y protagonistas de las propias evaluaciones, las cuales han de salir del espacio del aula para generar cambios y mejoras en las culturas inclusivas del propio centro escolar, así como en las políticas y prácticas. El concepto aparece en el Index for Inclusion (Booth y Ainscow, 2002).

Los resultados han coincidido también a la hora de indicar la necesidad de que el profesorado favorezca la creencia de que todo alumnado puede progresar al depositaren ellos altas expectativas, favoreciendo así su éxito (Arteaga y García-García, 2008; Liasidou y Antoniou, 2015). El resultado se relaciona con el concepto de transformabilidad, el cual indica que el profesorado ha de poseer concepciones sobre la educación inclusiva acordes con la capacidad de todo el alumnado por mejorar (Echeita, Simón, López y Urbina, 2013; Florian y Linklater, 2010).

Finalmente, se comparte la idea de que el aprendizaje conlleva la necesidad de comprometerse con la mejora profesional y personal constante (Echeita, 2015), fomentando así el aprendizaje a lo largo de la vida tal y como apunta la European Commission (2017) y Rodríguez (2019).

\section{CONCLUSIONES}

Se puede concluir que el perfil competencial en educación inclusiva requiere de una formación transversal en competencias relacionadas con el trabajo en equipo, el liderazgo, los valores y la ética, la pedagogía aplicada, la tecnología, la evaluación y las habilidades personales.

El perfil competencial resultante permite concluir que, dada la tipología de competencias finales obtenidas, se requiere para su formación de una práctica, la cual refuerce conocimientos, transfiera habilidades y solidifique actitudes y valores. Por este motivo, es necesario que las universidades piensen de manera estratégica que centros de prácticas van a ofrecer y como cooperar con ellos, creando así redes de mejora y aprendizaje continuado.

Se destaca como aporte de los propios resultados, la necesidad de una formación en competencias relacionadas con la capacidad de generar espacios físicos accesibles y compartir materiales y conocimiento entre los distintos agentes educativos, los cuales intervienen en los procesos inclusivos de los centros educativos mediante el uso de la tecnología.

Ha existido un debate entre las diferentes personas expertas sobre la atribución a la universidad de la formación de las competencias relacionadas con el ámbito personal. Un grupo de expertos mostró su desacuerdo argumentando que las competencias personales han de estar ya resueltas antes del acceso a los estudios universitarios. Este es un debate que abre la posibilidad a futuras líneas de investigación.

El estudio ha permitido delimitar de una manera más concreta cuál CEl (clasificadas en sus correspondientes ámbitos) tendría que dominar el alumnado de los grados de educación primaria de las universidades de Cataluña al finalizar sus estudios. Consultar a personas expertas, localizadas en la zona geográfica donde se ha desarrollado la investigación ha permitido profundizar y concretar las competencias, teniendo siempre en cuenta las referencias internacionales sobre la temática tratada.

Europa requiere de consenso y acuerdo sobre que competencias formativas en educación inclusiva deben aparecer en los planes formativos de los grados de magisterio. El nuevo modelo implementado en las universidades europeas: EEES, ha de dotar de homogeneidad ala formación, dejando espacio para las particularidades de cada territorio. La colaboración entre universidades, personas expertas, alumnado y personas profesionales se hace imprescindible para llegar a acuerdos coherentes y en sintonía entre el campo teórico y de conocimiento y el campo práctico-profesional. La cooperación fomenta, de la 
misma manera que lo hace la educación inclusiva, el crecimiento a partir del enfrentamiento de las realidades individuales con las colectivas, y genera aprendizajes significativos y transversales permanentes en el tiempo, dentro de la misma comunidad: la europea.

\section{REFERENCIAS}

Agency for Special Needs (2018). Pruebas de la relación entre la educación inclusiva y la inclusión social EUROPEAN AGENCY for Special Needs and Inclusive Education. Recuperado de www.europeanagency.org

Alegre de la Rosa, O. (2010). Capacidades docentes para atender la diversidad. Una propuesta vinculada a las necesidades básicas. Revista educación inclusiva, 186.

Arteaga, B. \& García-García, M. (2008). La formación de competencias docentes para incorporar estrategias adaptativas en el aula. Revista complutense de educación, 253-257. https://doi.org/10.5209/ RCED.16298

Blanco, R.\& Duk, C. (2019). El Legado de la Conferencia de Salamanca en el Pensamiento, Políticas y Prácticas de la Educación Inclusiva. Revista latinoamericana de educación inclusiva, 13(2), 25-43. https://doi.org/10.4067/s0718-73782019000200025

Booth, T. \& Ainscow, M. (2002). Index for inclusion: developing learning and participation in schools. Centre for Studies on Inclusive Education. https://doi.org/Furze, T. (2012). Review. Index for inclusion: developing learning and participation in schools. Educational Psychology in Practice, 445. Retrieved from http://dx.doi.org/10.1080/02667363.2012.693682

Cabero, J.\& Barroso, J. (2013). La utilización del juicio de experto para la evaluación de tic: El coeficiente de competencia experta. Bordon. https://doi.org/10.13042/brp.2013.65202

Cabero, J. e Infante, A. (2014). Empleo del método Delphi y su empleo en la investigación en comunicación y edu- cación. Edutec. Revista Electrónica de Tecnología Educativa, 48. Recuperado de http://goo.gl/zDnTm9

Cabero, J.\& Ruiz-Palmero, J. (2017). Las Tecnologías de la Información y Comunicación para la inclusión: reformulando la brecha digital. IJERI: International Journal of Educational Research and Innovation, 0(9), 16-30.

Comisión Europea (2017). Preparing teachers for diversity: the role of initial teacher education: final report to DG Education, Youth, Sport and Culture of the European Commission. Public Policy and Management Institute.https://doi.org/10.2766/637002

Comité sobre los Derechos de las Personas con Discapacidad. (2018). Advance unedited version. En CRPD. https://doi.org/CAT/C/MAR/CO/4

Darling-Hammond, L., \& Lieberman, A. (2013). Teacher education around the world: Changing policies and practices. En Teacher Education Around the World: Changing Policies and Practices. https://doi. org/10.4324/9780203817551

Duk, C. \& Murillo, F. J. (2016). Editorial: La Inclusión como Dilema. Revista Latinoamericana de Educación Inclusiva, 10(1), 11-14. Recuperado de www.rinace.net/rlei/

Echeita, G., Simón, C., López, M. y Urbina, C. (2013). Educación inclusiva. Sistemas de referencia, coordenadas y vórtices de un proceso dilemático. En M.A. Verdugo y R. Shalock (Coords.), Discapacidad e inclusión. Manual para la docencia (pp. 307-328). Salamanca: Amaru.

Echeita, G. (2015). Competencias esenciales en la formación inicial de un profesorado inclusivo. Un proyecto de la Agencia Europea para el desarrollo de las necesidades educativas especiales. Tendencias pedagógicas, (19), 7-24. 
Fernández, J.M. (2012). competencias docentes para la inclusión del alumnado universitario en el marco del Espacio Europeo de Educación Superior. Revista de la Educación Superior, XLI (2)(162), 9-24.

Fernández, J.M.(2013). Competencias docentes y educación inclusiva. Revista Electronica de Investigacion Educativa, 15(2), 82-99.

Florian, L. (2015). Inclusive Pedagogy: A transformative approach to individual differences but can it help reduce educational inequalities? Scottish Educational Review, 37(5), 5-14. https://doi.org/10.1 080/13540602.2012.709732

Forgiony-Santos, J. O. (2019). Análisis conceptual de las prácticas inclusivas en el aula, diversidad y convivencia escolar. Aibi revista de investigación, administración e ingeniería, 7, 36-40. https://doi. org/10.15649/2346030x.504

García García, M. (2005). Educación adaptativa y escuela inclusiva: una forma de atender las diferencias de todos los estudiantes - Dialnet. ISBN 84-205-4342-X, 3-32. Recuperado de https://dialnet.unirioja.es/servlet/articulo?codigo $=2090785$

García, C., Herrera-Seda, C. \& Vanegas, C. (2018). Competencias Docentes para una Pedagogía Inclusiva. Consideraciones a partir de la Experiencia con Formadores de Profesores Chilenos Teacher Competences to Inclusive Pedagogy. Considerations from Chilean Teacher Educators' Experiences. Revista Latinoamericana de Educación Inclusiva, 12(2), 149-167. https://doi. org/10.4067/S0718-73782018000200010

Generalitat de Catalunya, departament d'ensenyament.Ofensiva de país a favor de l'èxit escolar. Pla per a la reducció del fracàs escolar a Catalunya, 2012-2018). Abril 2013.

Gil-Gómez de Liaño, B., \& Pascual-Ezama, D. (2012). La metodología Delphi como técnica de estudio de la validez de contenido. Anales de Psicologia. https://doi.org/10.6018/analesps.28.3.156211

Grande, P., \& González, M. (2015). La educación inclusiva en la educación infantil: propuestas basadas en la evidencia. Tendencias pedagógicas, N 26(26), 145-162.

Hardy, I., \& Woodcock, S. (2015). Inclusive education policies: Discourses of difference, diversity and deficit. International Journal of Inclusive Education. https://doi.org/10.1080/13603116.2014.908965

Hung, H. L., Altschuld, J. W. \& Lee, Y. (2008). Methodological and concep- tual issues confronting a cross-country Delphi study of educational program evaluation. Evaluation and Program Plannin, 31, 191-198

Izuzquiza, D., Echeita, G., \& Simón, C. (2015). La percepción de estudiantes egresados de magisterio en la universidad autónoma de madrid sobre su competencia profesional para ser "profesorado inclusivo": un estudio preliminar. Tendencias pedagógicas.

J. Skulmoski, G., T. Hartman, F. \& Krahn, J. (2007). The Delphi Method for Graduate Research. Journal of Information Technology Education: Research, 6, 1-21. https://doi.org/10.28945/199

Jiménez Cortés, R. (2012). Educación inclusiva y formación inicial del profesorado: Evaluación de una innovación didáctica basada en la producción cinematográfica desde la perspectiva del alumnado. Revista de Educacion, (359), 232-259. https://doi.org/10.4438/1988-592X-RE-2011-359-093

Leiva J, J. \& Gómez Gerdel, M. (2015). La educación inclusiva como constructo pedagógico en el alumnado universitario de educación primaria. Revista nacional e internacional de educación inclusiva, Volumen 8, 185-200. Recuperado de http://revistaeducacioninclusiva.es/index.php/REl/article/ view/114

Liasidou, A., \& Antoniou, A. (2015). Head teachers leadership for social justice and inclusion. School Leadership and Management. https://doi.org/10.1080/13632434.2015.1010499

Luzón Trujillo, Antonio y Montes Moreno, Soledad. "Perspectiva histórica de la formación inicial del profesorado de Educación Infantil y Primaria en España. Una tarea inacabada", (Julio-Diciembre 2018) Vol. XIII No. 33: 119-150. DOI: http://dx.doi.org/10.15648/ hc.33.2018.6 
Mas, O. (2009). El perfil competencial del profesorado universitario en el Espacio Europeo de Educación Superior. Bellaterra: Departamento de Pedagogía Aplicada-UAB (Tesis Doctoral inédita).

Mas-Torelló, Ó., \& Olmos-Rueda, P. (2016). El profesor universitario en el espacio europeo de educación superior: La autopercepción de sus competencias docentes actuales y orientaciones para su formación pedagógica. Revista Mexicana de Investigacion Educativa.

Moriña, A., Cortés, M., \& Molina, V. (2015). Educación inclusiva en la enseñanza superior : soñando al profesorado ideal. Revista Latinoamericana de Educación Inclusiva, 9(2), 161-175.

Novo-Corti, l., Muñoz-Cantero, J. M., \& Calvo-Porral, C. (2011). Análisis de las actitudes de los jóvenes universitarios hacia la discapacidad: Un enfoque desde la teoría de la acción razonada. RELIEVE Revista Electronica de Investigacion y Evaluacion Educativa. https://doi.org/10.7203/relieve.17.2.4010

Perlado, I., Muñoz, Y., Torrego, J. (2019). Implicaciones de la formación del profesorado en aprendizaje cooperativo para la educación inclusiva. Profesorado. Revista de Currículum y Formación de Profesorado, 23(4), 128- 151. DOI: 10.30827/profesorado.v23i4.9468151

Prades, A. (2005). Resum sobre els enfocaments de competències. En AQU. Eines per l'adaptació dels ensenyaments a l'EEES. Barcelona: Agència Catalana per la Qualitat del Sistema Universitari de Catalunya.

Pujolàs, P. (2010). No es inclusión todo lo que se dice que lo es. Aula de innovación educativa, (191), 38-41.

Rodríguez, H. J. (2019). La Formación inicial del profesorado para la inclusión. Un urgente desafío que es necesario atender. Publicaciones, 49(3), 211-225. doi:10.30827/publicaciones.v49i3.11410

Sales Ciges, Auxiliadora (2006). Preservice teacher training for diversity: a methodological proposal for the new european higher education area. Revista interuniversitaria de formación del profesorado, 20, 201-217. Recuperat de http://www.redalyc.org/pdf/274/27411311010.pdf

Srivastava, M., de Boer, A., \& Pijl, S. J. (2015). Inclusive education in developing countries: a closer look at its implementation in the last 10 years. Educational Review. https://doi.org/10.1080/00131911.2 013.847061

Steurer , J. (2011). The Delphi method: an efficient procedure to generate knowledge. Skeletal Radiol, 40, 959- 961

UNESCO (2016). Training Tools for Curriculum Development. Reaching out to all Lear- ners: a Resource Pack Supporting Inclusive Education. Geneva: International Bureau of Education

Van Laarhoven, T. R., Munk, D. D., Lynch, K., Bosma, J., \& Rouse, J. (2007). A model for preparing special and general education preservice teachers for inclusive education. Journal of Teacher Education, 58(5), 440-455. https://doi.org/10.1177/0022487107306803

Para citar este documento:

Garcia-Vallès, X. (2020). Delimitación del perfil competencial inclusivo necesario en la formación inicial en los grados de educación primaria. Revista Innovaciones Educativas. 22(32), 51-64. DOI: https://doi.org/10.22458/ie.v22i32.2830 